\title{
The Gamow-Teller response in deformed nuclei
}

\author{
P. Sarriguren, R. Álvarez-Rodríguez, O. Moreno \\ Instituto de Estructura de la Materia, \\ Consejo Superior de Investigaciones Cientificas \\ Serrano 123, E-28006 Madrid, Spain \\ sarriguren@iem.cfmac.csic.es \\ E. Moya de Guerra \\ Departamento de Física Atómica, Molecular y Nuclear, Facultad de Ciencias Físicas, \\ Universidad Complutense de Madrid, E-28040 Madrid, Spain
}

\begin{abstract}
We study Gamow-Teller strength distributions within a QRPA approach performed on top of a deformed Skyrme-Hartree-Fock+BCS single particle basis with the inclusion of residual spin-isospin interactions in both particle-hole and particle-particle channels. We focus our attention in several problems of interest in Nuclear Structure and Nuclear Astrophysics, such as the $\beta$-decay properties of proton-rich medium-mass nuclei of astrophysical interest and the deformation dependence of the Gamow-Teller strength distributions in neutron deficient $\mathrm{Pb}$ isotopes. We also discuss the role of deformation in the two-neutrino double beta decay.
\end{abstract}

\section{INTRODUCTION}

The new experimental facilities involving radioactive ion beams provide us new information about the properties of nuclei with unusual proton to neutron ratios. This challenges the theoretical efforts to the description of these unusual objects. The decay properties and nuclear reactions of exotic nuclei are also essential ingredients to understand the late phases of the stellar life. Since most of the nuclear astrophysical input cannot be determined experimentally in the laboratory for the extreme conditions of temperature and density holding in the stellar medium, reliable theoretical calculations for these processes are absolutely necessary. As an example, the decay properties of proton rich nuclei are fundamental to understand the rapid proton capture processes (rp) occuring in X-ray bursts.

The theoretical framework used to describe these processes has been explained in detail elsewhere. [1] We construct first the quasiparticle basis selfconsistently from a deformed Hartree-Fock (HF) calculation with density-dependent Skyrme forces and pairing correlations. Then we solve the QRPA equations with separable residual interactions in both particle-hole $(p h)$ and particle-particle $(p p)$ channels.

In this work, we stress the role played by the nuclear deformation in various selected cases of special interest. We discuss first the results obtained in the iron mass region, comparing them with results from full shell model calculations, as well as with experiment. Then, we discuss the $\beta$-decay properties in a series of proton rich isotope chains approaching $N=Z$ in the mass region $A=70$, and in the neutron-deficient even isotopes ${ }^{184-194} \mathrm{~Pb}$. We discuss the sensitivity of our calculations to deformation, pairing, and residual interactions.

Finally, we apply the formalism to the simultaneous description of single beta Gamow-Teller strength distributions and to the two-neutrino double beta decay matrix elements in double beta emitters. We discuss the sensitivity of the double beta decay matrix elements to the deformed mean field and to the residual interactions. Nuclear deformation is found to be a mechanism of suppression in two-neutrino double beta decay.

\section{SKYRME HF+BCS+QRPA FORMALISM IN DEFORMED NUCLEI}

It is well known that the density-dependent HF approximation gives a very good description of ground-state properties for both spherical and deformed nuclei [2] and it is at present the most reliable mean field description.

The single-particle HF solutions for axially symmetric deformed nuclei are expanded into the eigenfunctions of an axially deformed harmonic-oscillator potential using eleven harmonic oscillator major shells $N$. Truncation effects are minimized by a proper choice of the oscillator parameters of the basis. We include pairing between like nucleons in the BCS approximation with fixed gap parameters for protons $\Delta_{\pi}$, and neutrons $\Delta_{\nu}$, which are determined phenomenologically from the odd-even mass differences.

To describe Gamow-Teller transitions we add to the mean field a spin-isospin residual interaction, which is expected to be the most relevant force for that purpose. This interaction contains two parts $p h$ and $p p$. The $p h$ part is responsible for the position and structure of the GT resonance $[1,3]$ and it may be derived consistently from the same energy density functional (and Skyrme interaction) as the HF equation, in terms of the second derivatives of the energy 
density functional with respect to the one-body densities. The $p h$ residual interaction is written in a separable form by averaging the Landau-Migdal resulting force over the nuclear volume, as explained in Refs. [1, 4]

$$
V_{G T}^{p h}=2 \chi_{G T}^{p h} \sum_{K=0, \pm 1}(-1)^{K} \beta_{K}^{+} \beta_{-K}^{-}, \quad \beta_{K}^{+}=\sum_{\pi \nu}\left\langle\nu\left|\sigma_{K}\right| \pi\right\rangle a_{\nu}^{+} a_{\pi} .
$$
$[1]$

The coupling strength $\chi_{G T}^{p h}$ is determined by the Skyrme parameters, the nuclear radius, and the Fermi momentum

$$
\chi_{G T}^{p h}=-\frac{3}{8 \pi R^{3}}\left\{t_{0}+\frac{1}{2} k_{F}^{2}\left(t_{1}-t_{2}\right)+\frac{1}{6} t_{3} \rho^{\alpha}\right\} .
$$

The particle-particle part is a neutron-proton pairing force in the $J^{\pi}=1^{+}$coupling channel. We introduce this interaction in the usual way, [3] that is, in terms of a separable force with a coupling constant $\kappa_{G T}^{p p}$, which is fitted to the phenomenology.

$$
V_{G T}^{p p}=-2 \kappa_{G T}^{p p} \sum_{K}(-1)^{K} P_{K}^{+} P_{-K}, \quad P_{K}^{+}=\sum_{\pi \nu}\left\langle\pi\left|\left(\sigma_{K}\right)^{+}\right| \nu\right\rangle a_{\nu}^{+} a_{\bar{\pi}}^{+} .
$$

The proton-neutron QRPA phonon operator for GT excitations in even-even nuclei is written as

$$
\Gamma_{\omega_{K}}^{+}=\sum_{\pi \nu}\left[X_{\pi \nu}^{\omega_{K}} \alpha_{\nu}^{+} \alpha_{\overline{\bar{\pi}}}^{+}+Y_{\pi \nu}^{\omega_{K}} \alpha_{\bar{\nu}} \alpha_{\pi}\right],
$$

where $\omega_{K}$ are the excitation energies, and $X_{\pi \nu}^{\omega_{K}}, Y_{\pi \nu}^{\omega_{K}}$ the forward and backward amplitudes, respectively. They are obtained by solving the QRPA equations. For even-even nuclei the GT transition amplitudes in the intrinsic frame connecting the QRPA ground state $|0\rangle\left[\Gamma_{\omega_{K}}|0\rangle=0\right]$ to one phonon states $\left|\omega_{K}\right\rangle\left[\Gamma_{\omega_{K}}^{+}|0\rangle=\left|\omega_{K}\right\rangle\right]$, are given by

$$
\left\langle\omega_{K}\left|\beta_{K}^{ \pm}\right| 0\right\rangle=\mp M_{ \pm}^{\omega_{K}}
$$

with

$$
\begin{gathered}
M_{-}^{\omega_{K}}=\sum_{\pi \nu}\left(q_{\pi \nu} X_{\pi \nu}^{\omega_{K}}+\tilde{q}_{\pi \nu} Y_{\pi \nu}^{\omega_{K}}\right), \quad M_{+}^{\omega_{K}}=\sum_{\pi \nu}\left(\tilde{q}_{\pi \nu} X_{\pi \nu}^{\omega_{K}}+q_{\pi \nu} Y_{\pi \nu}^{\omega_{K}}\right), \\
\tilde{q}_{\pi \nu}=u_{\nu} v_{\pi} \Sigma_{K}^{\nu \pi}, \quad q_{\pi \nu}=v_{\nu} u_{\pi} \Sigma_{K}^{\nu \pi},
\end{gathered}
$$

$v^{\prime}$ s are occupation amplitudes $\left(u^{2}=1-v^{2}\right)$ and $\Sigma_{K}^{\nu \pi}$ spin matrix elements connecting neutron and proton states with spin operators

$$
\Sigma_{K}^{\nu \pi}=\left\langle\nu\left|\sigma_{K}\right| \pi\right\rangle .
$$

The total half-life $T_{1 / 2}$ for allowed $\beta$ decay from the ground state of the parent nucleus is obtained by summing the strengths over all the final states involved in the process with excitation energy below the $Q$-value,

$$
T_{1 / 2}^{-1}=\frac{\kappa^{2}}{6200} \sum_{\omega} f(Z, \omega)\left|\left\langle 1_{\omega}^{+}\left\|\beta^{+}\right\| 0^{+}\right\rangle\right|^{2},
$$

where $f(Z, \omega)$ is a phase space factor. We include effective factors

$$
\kappa^{2}=\left[\left(g_{A} / g_{V}\right)_{\mathrm{eff}}\right]^{2}=\left[0.77\left(g_{A} / g_{V}\right)_{\mathrm{free}}\right]^{2} .
$$


Once the intrinsic amplitudes $\left\langle f\left|\beta_{K}^{ \pm}\right| i\right\rangle$ are calculated, the Gamow-Teller strength $B(G T)$ in the laboratory system for a transition $I_{i} \rightarrow I_{f}$ can be obtained as

$$
B\left(G T^{ \pm}\right)=\sum_{M_{i}, M_{f}, \mu}\left|\left\langle I_{f} M_{f}\left|\beta_{\mu}^{ \pm}\right| I_{i} M_{i}\right\rangle\right|^{2}
$$

in units of $g_{A}^{2} / 4 \pi$. The initial and final states in the laboratory frame are expressed in terms of the intrinsic states $\left|\phi_{K}\right\rangle$ using the Bohr-Mottelson factorization. In the case of even-even parent nuclei, $I_{i}=K_{i}=0, I_{f}=1$, and $K_{f}=0,1$, we obtain

$$
B\left(G T^{ \pm}\right)=\frac{g_{A}^{2}}{4 \pi}\left\{\delta_{K_{f}, 0}\left\langle\phi_{K_{f}}\left|\beta_{0}^{ \pm}\right| \phi_{0}\right\rangle^{2}+2 \delta_{K_{f}, 1}\left\langle\phi_{K_{f}}\left|\beta_{1}^{ \pm}\right| \phi_{0}\right\rangle^{2}\right\}
$$

\section{GAMOW-TELLER STRENGTH DISTRIBUTIONS}

We investigate first the GT properties of nuclei in the region of medium masses around $\mathrm{A}=56$, which are of special importance because they are the main constituents of the stellar core in presupernovae formations. In the last decades, $\mathrm{GT}_{+}$strength distributions in this mass region have been studied experimentally via $(n, p)$ charge exchange reactions at forward angles. As a result, a large amount of experimental information is available to compare with. We find that the present pnQRPA calculations are able to reproduce the main features of the GT properties in this mass region, [5] reinforcing the confidence in the method and in its predictive power. Although in this mass region the full shell model calculations of Ref. [6] may be superior, and some fine details of data may be better described, we find that on the overall the agreement with experiment of present pnQRPA calculations is comparable. In addition, our approach can be extended to much heavier nuclei beyond the present capability of the full shell model, without increasing the complexity of the calculations.

To study shape coexistence one has to perform constrained HF calculations. In this way, minimization of the HF energy under the constraint of holding the nuclear deformation fixed is carried out over a range of deformations. When more than one local minimum occurs for the total energy as a function of deformation, shape coexistence results. This type of constrained calculations has been done in Ref. [1] with the result that most neutron-deficient Ge, Se, Kr, and Sr isotopes are candidates for shape coexistence.
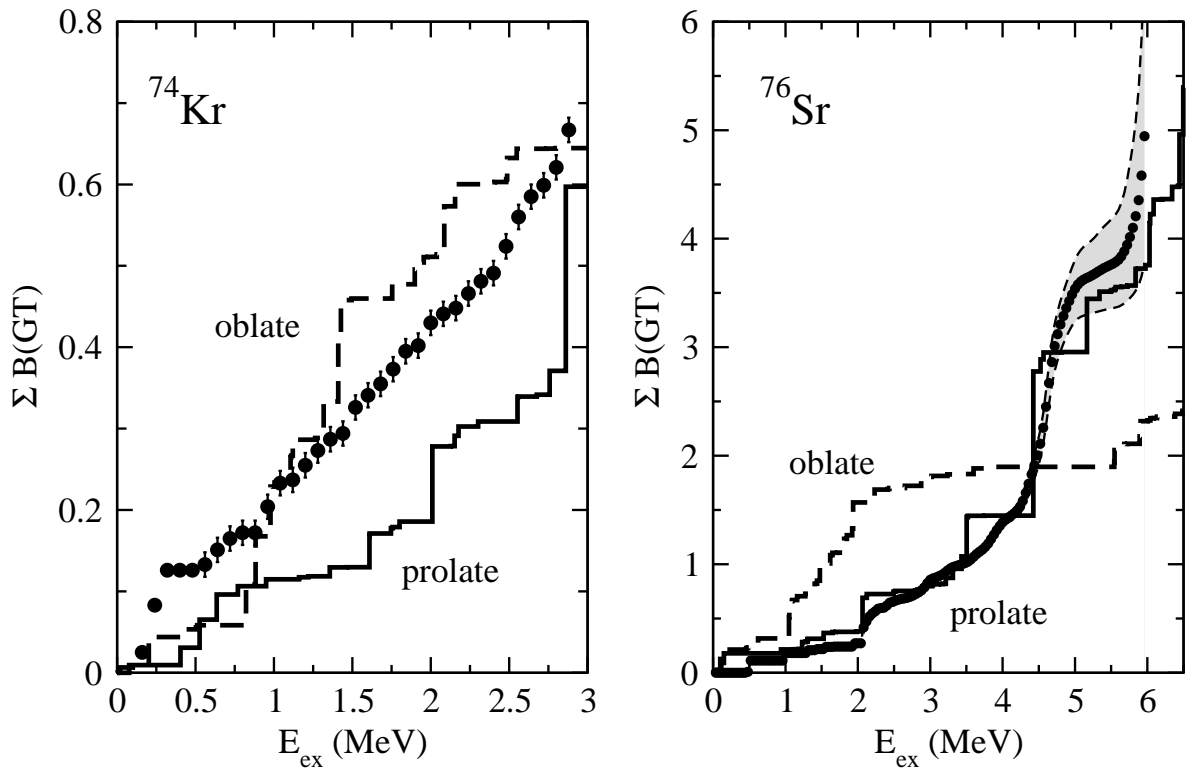

FIG. 1: Accumulated GT strength in ${ }^{74} \mathrm{Kr}$ (left) and ${ }^{76} \mathrm{Sr}$ (right) as a function of the energy of the daughter nucleus. Experimental data are compared with the theoretical calculations assuming prolate (solid lines) and oblate (dashed lines) shapes for the ground state.

The sensitivity of the GT strength distributions to the deformation of the decaying nucleus has been exploited as an alternative tool to extract information on the shape of exotic nuclei. Fig. 1 shows the accumulated GT strength 
distributions in ${ }^{74} \mathrm{Kr}$ and ${ }^{76} \mathrm{Sr}$ calculated for both oblate and prolate equilibrium shapes. They are compared to recent experimental data from CERN-ISOLDE. [7] This comparison shows that while the data are incompatible with an oblate shape and favors strongly a prolate ground state in the case of ${ }^{76} \mathrm{Sr}$, in the case of ${ }^{74} \mathrm{Kr}$ the data indicate an oblate/prolate shape mixing.

Many problems in Nuclear Astrophysics usually require the use of numerical simulations and network calculations using weak interaction rates as input parameters. High quality nuclear input is therefore a necessary condition for a high quality astrophysical model. In particular, the rapid proton capture (rp) process is of special interest. [8] It is expected to take place in explosive scenarios, such as X-ray bursts, where the necessary conditions of high densities and temperatures are met. The rp-process is characterized by the fact that the proton capture reaction rates are orders of magnitude faster than the competing $\beta^{+}$decays. When the proton capture is inhibited, the reaction flow has to wait for a relatively slow $\beta^{+}$decay to continue. The longer lived $\beta^{+}$emitters in this process are called waiting points. The half-lives of the waiting point nuclei determine the time scales of the flow.

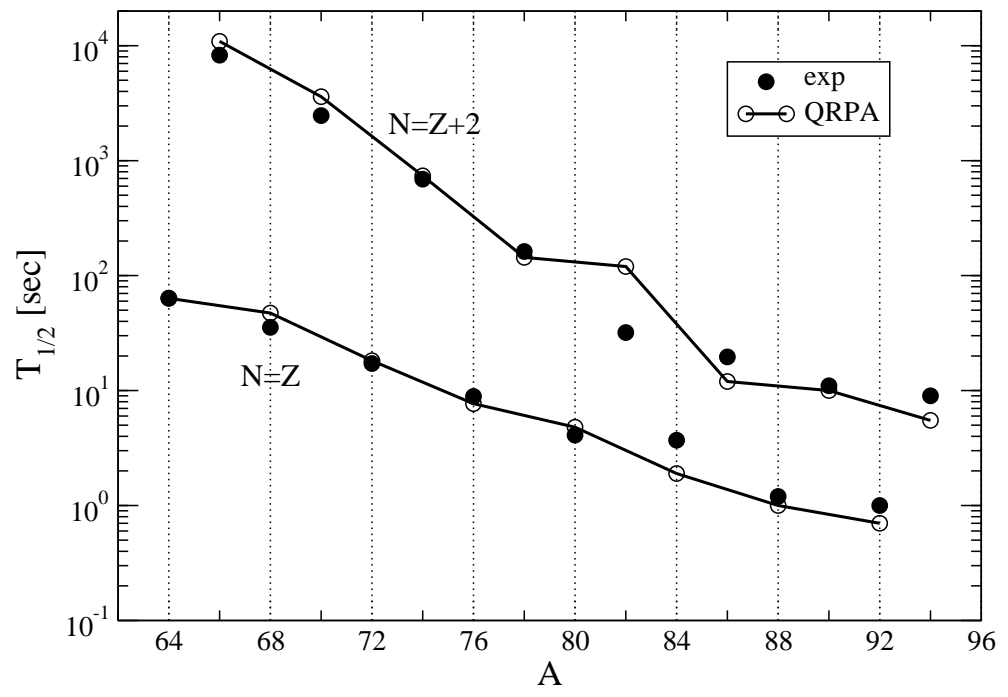

FIG. 2: Half-lives of the $N=Z$ waiting points $(\mathrm{A}=64-92)$ and $N=Z+2$ obtained within the QRPA formalism compared to experiment.

We have calculated the half-lives of waiting point nuclei in the mass region $A=60-90$ within the QRPA formalism. [9] Analyzing the half-lives as a function of deformation, we find that the best agreement with the experimental halflives is obtained using the selfconsistent deformations obtained from the minimization of the energy. Fig. 2 shows the QRPA results for the half-lives of the $\mathrm{N}=\mathrm{Z}$ waiting points and their $\mathrm{N}=\mathrm{Z}+2$ neighbors. The results obtained indicate that this formalism is a useful method for reliable calculations of half-lives, which is especially interesting for applications to cases where no experimental information is available and to nuclei that are beyond the capability of full shell model calculations.

This type of analysis has been recently extended to the study of the GT strength distributions in the neutrondeficient even isotopes ${ }^{184-194} \mathrm{~Pb}$, where the phenomenon of shape coexistence has been experimentally observed. [10] By analyzing the sensitivity of the GT strength distributions to the various ingredients in the formalism, we conclude that the $\beta$-decay of these isotopes could be a useful tool to look for fingerprints of nuclear deformation. [11]

Fig. 3 shows the results obtained from a constrained HF calculation in this mass region and Fig. 4 shows the GT strength distributions calculated at the equilibrium shapes for the case of ${ }^{194} \mathrm{~Pb}$. By investigating the sensitivity of our results to the forces used, we arrive to the conclusion that the energy deformation curves are very sensitive to the Skyrme and pairing forces used. The ground state shape predicted may be modified with different choices for these forces. Nevertheless, the qualitative behavior of the energy profile is robust in the sense that the deformations at which the minima occur are hardly shifted and that the shape coexistence between spherical, oblate and prolate shapes is a constant feature in these isotopes. We also observe that the GT strength distributions show specific characteristics for each deformation that remain against changes of the Skyrme and pairing forces. The effect of the deformation on the GT strength distributions is much stronger than the effects coming from the Skyrme or pairing forces used. As a consequence, we identify in Fig. 4 clear signatures of deformation related to the profiles of the GT strength distributions, which are peaked at different energies depending on the shape of the decaying nucleus. 


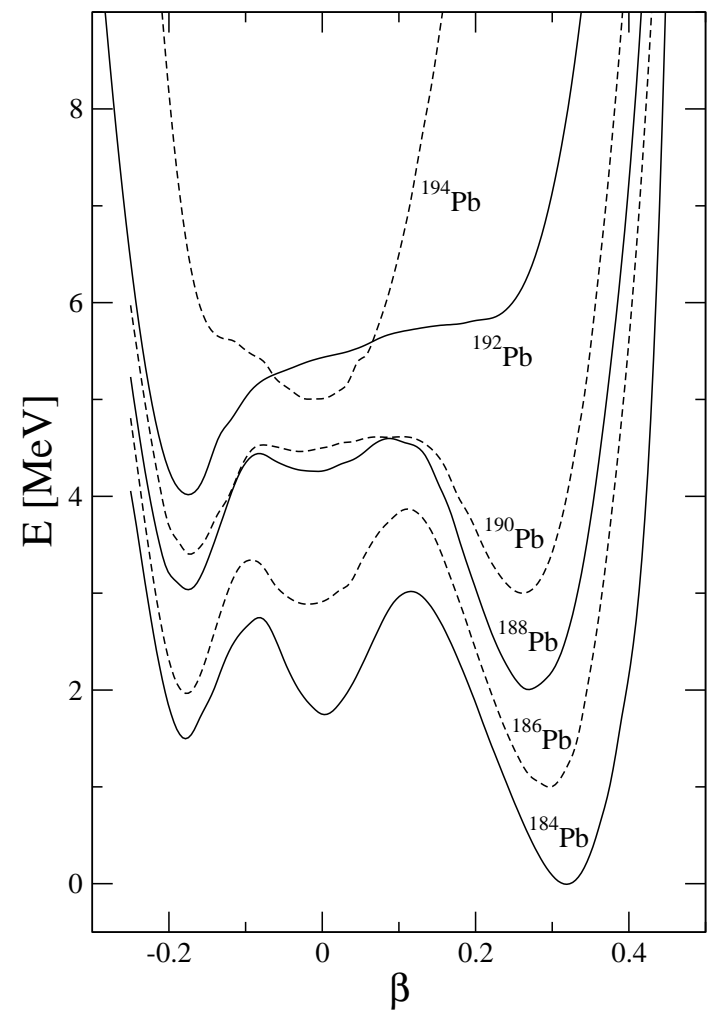

FIG. 3: HF energy of the ${ }^{184,186,188,190,192,194} \mathrm{~Pb}$ isotopes as a function of the quadrupole deformation parameter $\beta$. The results correspond to a constrained HF+BCS calculation with the Skyrme interaction Sk3 and fixed gap parameters.
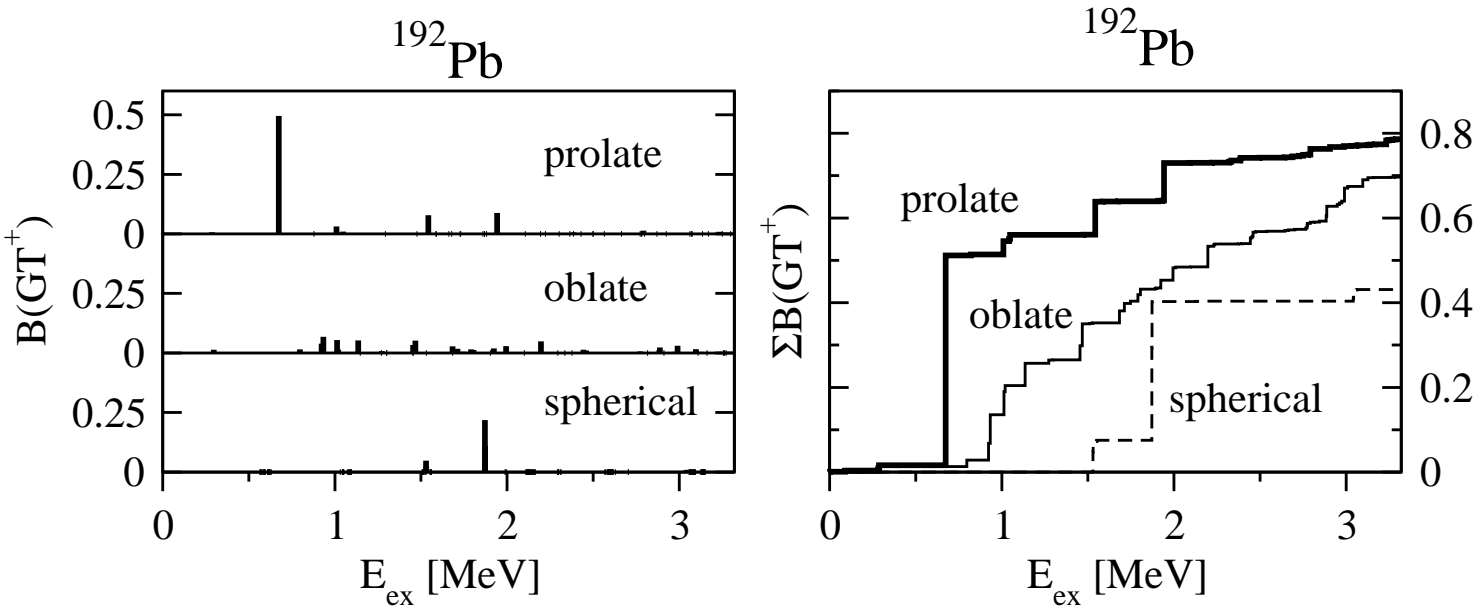

FIG. 4: GT strength distributions in the ${ }^{192} \mathrm{~Pb}$ isotopes for spherical, oblate and prolate shapes. Results are obtained from the force SLy4 with fixed gap parameters. The right panel shows the accumumlated $B\left(G T^{+}\right)$.

\section{TWO-NEUTRINO DOUBLE BETA DECAY}

Nuclear double beta decay is a rare second order weak interaction process that takes place when the transition to the intermediate nucleus is energetically forbidden or highly retarded. Two main decay modes are expected in this process. The two neutrino mode, involving the emission of two electrons and two neutrinos, and the neutrinoless mode with no neutrino leaving the nucleus. While the first type of process is perfectly compatible with the Standard Model, the second one violates lepton number conservation and its observation is linked to the existence of a massive Majorana neutrino. For this reason, considerable experimental and theoretical effort is being devoted to the study of this process. [12] 
From the theoretical point of view, one particular source of uncertainty is the evaluation of the nuclear matrix elements involved in the process. They have to be calculated as accurately as possible to obtain reliable estimates for the limits of the double beta decay half-lives. Since the nuclear wave functions and the underlying theory for treating the neutrinoless and the two-neutrino modes are similar, the usual procedure is to test first the nuclear structure component of the two-neutrino mode against the available experimental information on half-lives.

We use a deformed QRPA formalism to describe simultaneously the energy distributions of the single beta GamowTeller strength that build up the double beta process and the two-neutrino double beta decay $(2 \nu \beta \beta)$ matrix elements. [13] Calculations are performed using deformed Skyrme Hartree-Fock mean fields. The deformation is determined selfconsistently and good agreement with experimental charge radii and quadrupole moments is obtained.

The $2 \nu \beta \beta$ decay is described in second order perturbation of the weak interaction as two successive Gamow-Teller transitions via virtual intermediate $1^{+}$states. The half-life of the $2 \nu \beta \beta$ decay

$$
T_{1 / 2}^{2 \nu}\left(0_{\mathrm{gs}}^{+} \rightarrow 0_{\mathrm{gs}}^{+}\right)=\left[G^{2 \nu}\left|M_{\mathrm{GT}}^{2 \nu}\right|^{2}\right]^{-1}
$$

is given as a product of a phase space integral $G^{2 \nu}$ and the Gamow-Teller transition matrix element $M_{\mathrm{GT}}^{2 \nu}$, which contains the nuclear structure effects. For a transition connecting initial and final ground states, it is given by

$$
M_{\mathrm{GT}}^{2 \nu}=\sum_{K} \sum_{m_{i}, m_{f}} \frac{\left\langle 0_{f}^{+}\left\|\sigma_{K} t^{-}\right\| \omega_{K}^{m_{f}}\right\rangle\left\langle\omega_{K}^{m_{f}} \mid \omega_{K}^{m_{i}}\right\rangle\left\langle\omega_{K}^{m_{i}}\left\|\sigma_{K} t^{-}\right\| 0_{i}^{+}\right\rangle}{\left(\omega_{K}^{m_{f}}+\omega_{K}^{m_{i}}\right) / 2}
$$

where $K=0, \pm 1$ and $m_{i}, m_{f}$ label the number of intermediate $1^{+}$RPA states $\omega_{K}^{m_{i}}, \omega_{K}^{m_{f}}$ reached from the initial $\mid 0_{i}^{+}>$and final $\mid 0_{f}^{+}>$nuclear ground states, respectively. The overlap $<\omega_{K}^{m_{f}} \mid \omega_{K}^{m_{i}}>$ is needed to take into account the non-orthogonality of the intermediate states reached from the initial ground state to those reached from the final ground state.

We discuss the sensitivity of the parent and daughter Gamow-Teller strength distributions in single beta decay, as well as the sensitivity of the double beta decay matrix elements to the deformed mean field and to the residual interactions.

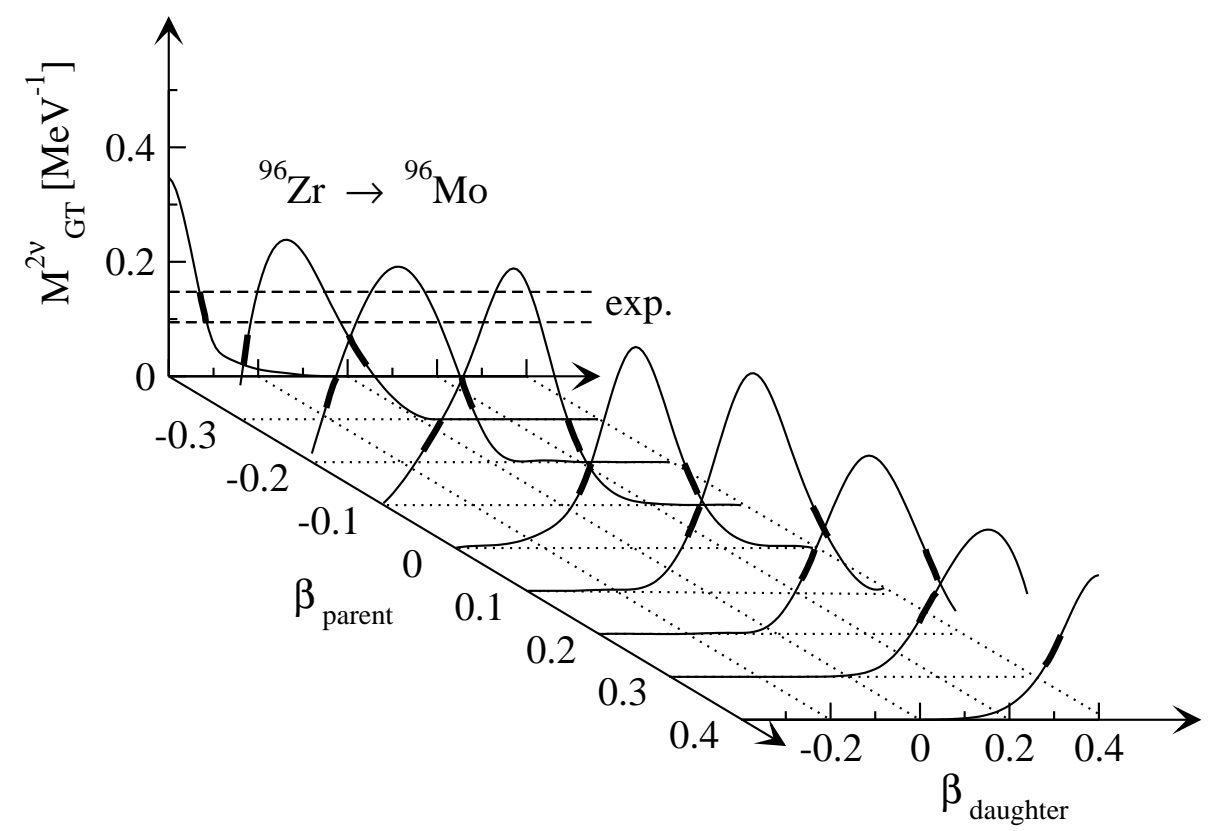

FIG. 5: $2 \nu \beta \beta$-decay matrix elements of ${ }^{96} \mathrm{Zr}$ obtained from Skyrme Hartree-Fock calculations as a function of both parent and daughter deformations.

Nuclear deformation is found to be a mechanism of suppression of the two-neutrino double beta decay. [13] The double beta decay matrix elements are found to have maximum values for about equal deformations of parent and daughter nuclei. They decrease rapidly when differences in deformations increase.

As an example of our results we show in Fig. 7 the single $\beta$-decay branches $\left(\beta^{-}\right.$in ${ }^{150} \mathrm{Nd}$ and $\beta^{+}$in $\left.{ }^{150} \mathrm{Sm}\right)$, as well as the $2 \nu \beta \beta$-decay matrix elements of ${ }^{150} \mathrm{Nd}$ as a function of the particle-particle interaction strength $\kappa_{p p}$. 

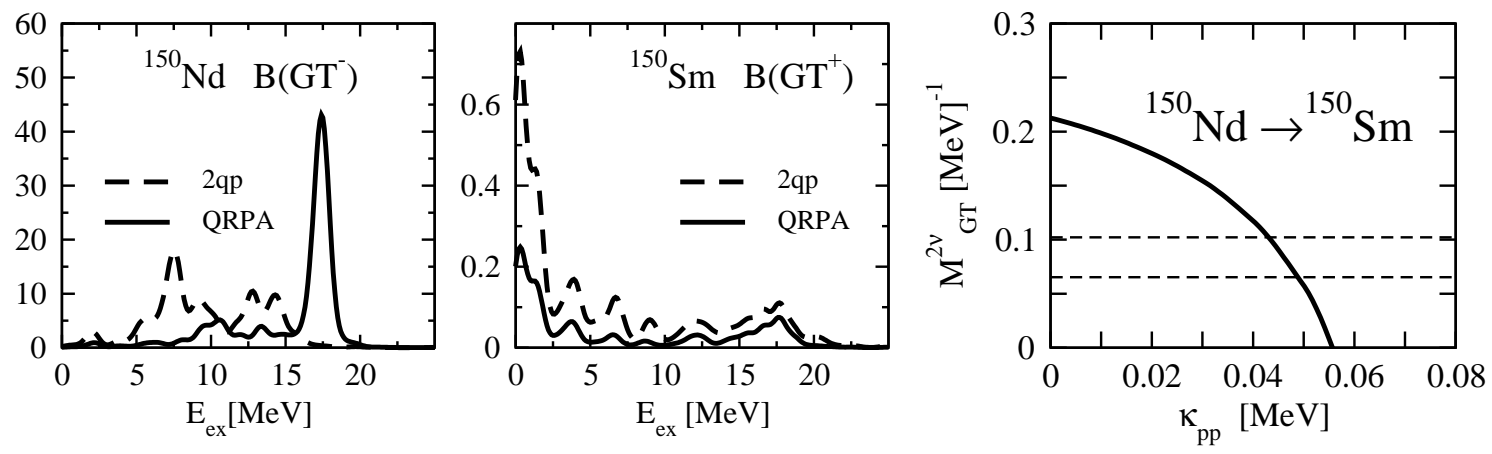

FIG. 6: Left: Gamow-Teller $B\left(G T^{-}\right)$strength distribution in ${ }^{150} \mathrm{Nd}$ as a function of the excitation energy in the daughter nucleus. Middle: $B\left(G T^{+}\right)$in ${ }^{150} \mathrm{Sm}$. Right: $2 \nu \beta \beta$-decay matrix elements of ${ }^{150} \mathrm{Nd}$ obtained from Skyrme Hartree-Fock calculations as a function of the $p p$ interaction strength $\kappa_{p p}$.

\section{CONCLUSIONS}

We have applied a deformed HF+BCS+QRPA formalism with density-dependent two-body effective Skyrme interactions to the description of $\beta$-decay properties in various isotopic chains of medium-mass proton-rich nuclei, as well as to the study of Gamow-Teller strength distributions in the iron and lead mass regions.

Very reasonable agreement with ground state, $\beta$-decay properties, as well as with GT and spin $M 1$ strength distributions is obtained. In the iron mass region, the comparison of pnQRPA results to data on GT strength distributions provides a fairly sound basis to safely apply this method to the estimates of GT strengths and particularly of $\beta$-decay properties of highly unstable nuclei in other mass regions. Though the GT strength distributions obtained from full shell model calculations may agree better with experiment in some details, the present HF+BCS+pnQRPA results are, on the overall, of comparable quality.

In the $A \sim 70$ mass region we have found shape isomerism in most of the isotopes studied and a nice agreement with available experimental data on $Q_{E C}$ values, half-lives, and GT strength distributions. From our study of the dependence on the shape of the GT strength distributions we conclude that information on the shape of the parent nucleus can be gained by comparison with experiment in the whole $Q_{E C}$ window.

The half-lives of $\mathrm{N}=\mathrm{Z}$ waiting point nuclei of relevance in the rp-process have been calculated within the QRPA formalism and analyzed as a function of deformation. The agreement of the results obtained with the available experimental information indicates that the formalism is useful to extrapolate to regions unexplored and to different conditions of densities and temperatures.

The effect of deformation on the $2 \nu \beta \beta$-decay matrix elements has been studied first by considering the deformations of both parent and daughter as free parameters. It is found that the matrix elements are suppressed with respect to the spherical case. More precisely, it is found a sizable reduction effect that scales with the deformation difference between parent and daughter. This suppression mechanism, which is ignored in spherical treatments, may play an important role in approaching the theoretical estimates to experiment.

\section{Acknowledgements}

This work was supported by Ministerio de Educación y Ciencia (Spain) under contract number FIS2005-00640.

[1] P. Sarriguren et al., Nucl. Phys. A635 (1998) 55; A658 (1999) 13; Nucl. Phys. A691 (2001) 631; Phys. Rev. C64 (2001) 064306.

[2] D. Vautherin and D. M. Brink, Phys. Rev. C5 (1972) 626; D. Vautherin, Phys. Rev. C7 (1973) 296; P. Bonche et al., Nucl. Phys. A443 (1985) 39.

[3] M. Hirsch et al., Nucl. Phys. A535, 62 (1991; K. Muto et al., Z. Phys. A341, 407 (1992).

[4] P. Sarriguren, E. Moya de Guerra and R. Nojarov, Phys. Rev. C54, 690 (1996); Z. Phys. A357, 143 (1997).

[5] P. Sarriguren et al., Nucl. Phys. A716 (2003) 230.

[6] E. Caurier et al., Nucl. Phys. A653 (1999) 439.

[7] E. Poirier et al., Phys. Rev. C69, 034307 (2004); E. Nacher et al., Phys. Rev. Lett. 92, 232501 (2004). 
[8] H. Schatz et al., Phys. Rep. 294, 167 (1998).

[9] P. Sarriguren et al., Eur. Phys. J. A24, 193 (2005).

[10] A.N. Andreyev et al., Nature 405, 430 (2000).

[11] P. Sarriguren et al., Phys. Rev. C72, 054317; O. Moreno et al., Phys. Rev. C73, 054302.

[12] J. Suhonen and O. Civitarese, Phys. Rep. 300 (1998) 123; A. Faessler and F. Šimkovic, J. Phys. G: Nucl. Part. Phys. 24 (1998) 2139; H.V. Klapdor-Kleingrothaus, Sixty Years of Double Beta Decay, World Scientific, Singapore (2001).

[13] R. Álvarez-Rodríguez et al., Phys. Rev. C70, 064309 (2004). 